\title{
Catalan lattices on series parallel interval orders
}

\author{
Filippo Disanto* $\quad$ Luca Ferrari $^{\dagger} \quad$ Renzo Pinzani ${ }^{\dagger}$ \\ Simone Rinaldi*
}

\begin{abstract}
Using the notion of series parallel interval order, we propose a unified setting to describe Dyck lattices and Tamari lattices (two well known lattice structures on Catalan objects) in terms of basic notions of the theory of posets. As a consequence of our approach, we find an extremely simple proof of the fact that the Dyck order is a refinement of the Tamari one. Moreover, we provide a description of both the weak and the strong Bruhat order on 312-avoiding permutations, by recovering the proof of the fact that they are isomorphic to the Tamari and the Dyck order, respectively; our proof, which simplifies the existing ones, relies on our results on series parallel interval orders.
\end{abstract}

\section{Introduction}

The set of all Dyck paths of fixed length can be ordered by setting $P \leq Q$ when $P$ lies weakly below $Q$ (in the usual two-dimensional drawings of Dyck paths). This partial order is in fact a lattice, called Dyck lattice. We point out that this structure is not new and, in some sources, it is called Stanley lattice [4, 13. The Hasse diagram of the Dyck lattice on the set of Dyck paths of length 6 is represented in figure 1(a).

It is well known that Dyck paths of length $2 n$ are counted by the $n$-th Catalan number $C_{n}=\frac{1}{n+1}\left(\begin{array}{c}2 n \\ n\end{array}\right)$. The different incarnations of the Catalan family give rise to several further lattices beside Dyck's. Among them, the Tamari lattice is indeed one of the more widely known, and appears naturally in the study of binary trees and of the Stasheff polytope [11. See figure 1(b) for the Hasse diagram of the Tamari lattice with five elements.

Using suitable bijections between Dyck paths, binary trees and planar trees, the two mentioned Catalan lattices can be defined on the set of plane trees of size $n$ in such a way that the Dyck lattice with $n$ elements is an extension of the Tamari lattice with $n$ elements (see [13]).

\footnotetext{
* Dipartimento di Scienze Matematiche ed Informatiche, Pian dei Mantellini, 44, 53100, Siena, Italy disafili@yahoo.it rinaldi@unisi.it

${ }^{\dagger}$ Dipartimento di Sistemi e Informatica, viale Morgagni 65, 50134 Firenze, Italy ferrari@dsi.unifi.it pinzani@dsi.unifi.it
} 


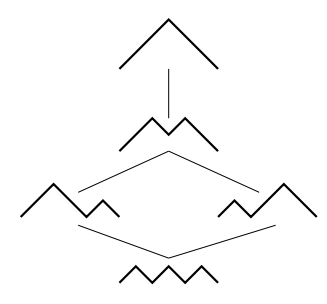

(a)

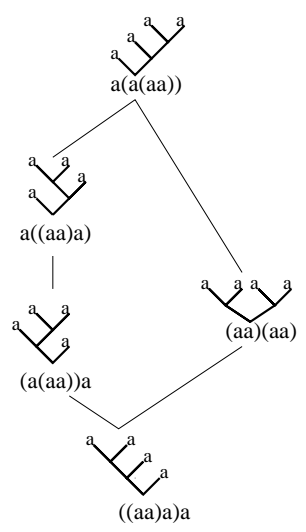

(b)

Figure 1: (a) The Dyck lattice with five elements; (b) The Tamari lattice with five elements.

In this paper, we will consider yet another occurrence of Catalan structures, the so called series parallel interval orders. Our aim is to define the two above Catalan lattices on the set of series parallel interval orders with the aid of a special kind of linear extension. We propose this unified interpretation since, in our opinion, it allows to better understand the connections between Dyck and Tamari lattices. To obtain our characterization of the Dyck and Tamari lattices we will make use of some basic notions of the theory of posets. The known relationship between the two lattices will be quite simple to achieve in our setting.

Thanks to this approach we will also be able to provide a link between the Dyck (respectively, Tamari) lattice and the strong (respectively, weak) Bruhat order, when the latter is considered on the class of permutations avoiding the pattern 312 .

As already recalled, the main combinatorial objects in this approach are series parallel interval orders, which are the intersection of two important classes of partially ordered sets, namely series parallel orders [16] and interval orders [5, 10. Our approach will express important features of series parallel interval orders and so their use in this unified version of the two Catalan lattices seems to be relevant in its own.

\section{Series-parallel interval orders}

In this section we will focus on those posets having no induced subposet isomorphic either to the poset $2+2$ or to the fence of order four, shown in figure 
2. These partial orders are called series parallel interval orders. We will denote by $\mathcal{O}$ the class of such posets, also writing $\mathcal{O}(n)$ for those having precisely $n$ elements. We also warn the reader that, due to technical reasons, in what follows we will rather deal with the strict order relation associated with a series parallel interval order. Nevertheless, with an abuse of notation, we will always use the expression $R \in \mathcal{O}$ to mean that $R$ is the strict order relation associated with a series parallel interval order.

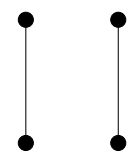

(a)

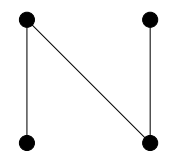

(b)

Figure 2: (a) The poset $2+2$; (b) The fence of order four.

This kind of posets has been recently considered in [7, where the authors show that they are enumerated by Catalan numbers according to the number of their elements; some bijections with other structures enumerated by Catalan numbers are also established. For our purposes, we need to recall here a bijection $\rho$ (stated in [7]) between planar trees with $n+1$ nodes and $\mathcal{O}(n)$. Given any planar tree $T$, we define the binary relation $R=\rho(T)$ on the set of its nodes other than the root, by setting $x R y$ whenever $x$ and $y$ cannot be joined by a directed path in $T$ (in the directed graph canonically determined by $T$ ) and $x$ lies on the left of $y$ in $T$. The resulting poset is indeed in $\mathcal{O}$. In figure 3 we can see an instance of the bijection $\rho$. In what follows, we will always represent rooted trees with the root at the bottom.
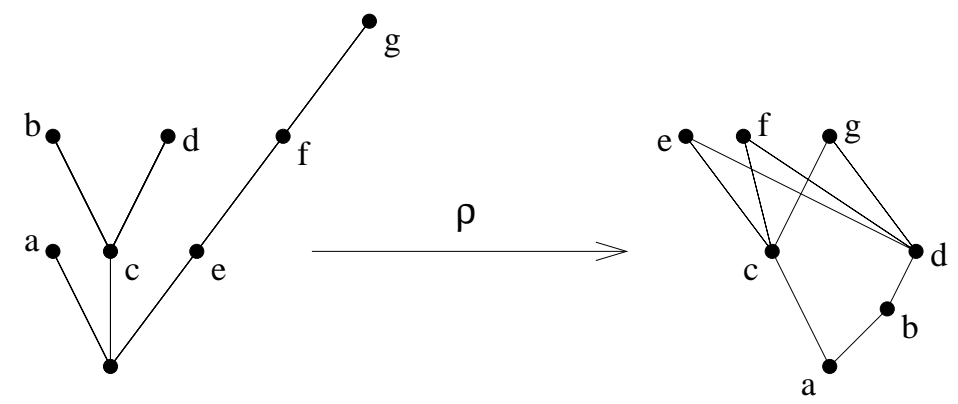

Figure 3: The bijection $\rho$ : a planar tree and the associated poset in $\mathcal{O}$. 


\subsection{Preorder linear extensions of series parallel interval orders}

In this section we will define a particular type of linear extension for the posets in $\mathcal{O}$ corresponding to the preorder traversal in the associated planar tree; for this reason we will call it the preorder linear extension.

In order to define the preorder linear extension of $R \in \mathcal{O}$, we need to define an auxiliary binary relation $Z(R)=Z$ on the support of $R$. Given a binary relation $B$, we set $\bar{B}=B \cup B^{-1}$ and we use the notation $B^{c}$ to indicate the complement of $B$. Now define $Z=\left((\bar{R})^{c} \circ \bar{R}\right) \backslash \bar{R}$. Recall that, for any two binary relations $X$ and $Y$ defined on the same set, the composition $X \circ Y$ is defined by setting $x(X \circ Y) y$ when there exists an element $z$ such that $x X z$ and $z Y y$ (see, for instance, [15]). Thus, we can rephrase the above definition by saying that $x Z y$ whenever $x \bar{R} y$ and there exists $z$ such that $z \bar{R} y$ and $z \bar{R} x$. Given $R \in \mathcal{O}$, if $x, y, z$ are such that $z R y$ and $x$ is incomparable with both $z$ and $y$, then $Z$ can be described as illustrated in figure 4 .
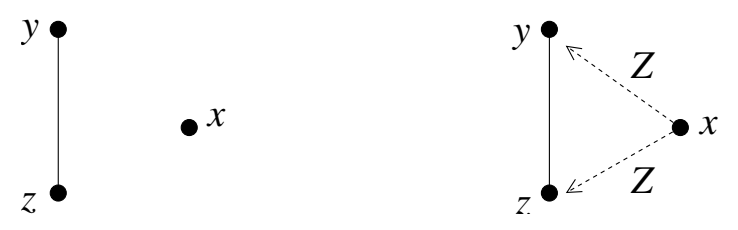

Figure 4: The relation $Z$ on an instance of the poset $2+1$.

We say that a linear extension $\lambda$ of $R$ is a preorder linear extension of $R$ when $x Z y$ implies $x \lambda y$. Figure 5 depicts a poset $R \in \mathcal{O}$, the relation $Z(R)$ and an associated preorder linear extension.

The next proposition shows that, for any $R \in \mathcal{O}$, there exists at most one preorder linear extension of $R$ up to (order) automorphisms. The proposition needs a preliminary lemma. In what follows, we will say that two elements of a poset are order equivalent when there exists an order automorphism mapping one of them into the other.

Lemma 2.1 Let $R \in \mathcal{O}$ and suppose that $\lambda_{1}$ and $\lambda_{2}$ are two preorder linear extensions of $R$. For any $x, y$ in the support of $R$, if $\lambda_{1}(x)>\lambda_{1}(y)$ and $\lambda_{2}(x)<$ $\lambda_{2}(y)$, then $x$ and $y$ must be order equivalent in $R$.

Proof. If $\lambda_{1}(x)>\lambda_{1}(y)$ then $(x, y) \notin R \cup Z$ and by $\lambda_{2}(x)<\lambda_{2}(y)$ follows that $(y, x) \notin R \cup Z$. From the definition of $Z$, this implies that, for every $z$, $z \bar{R} y$ or $z \bar{R} x$, and also that, for every $z, z \bar{R} y$ or $z \bar{R} x$. Equivalently, for every $z$, we get that either $z \bar{R} y$ and $z \bar{R} x$, or $z \bar{R} y$ and $z \bar{R} x$. Since $x \overline{R R} y$, it is now easy to show that, for every $a \neq x, y, a R x$ if and only if $a R y$ and $x R a$ if and only if $y R a$, whence the thesis. 


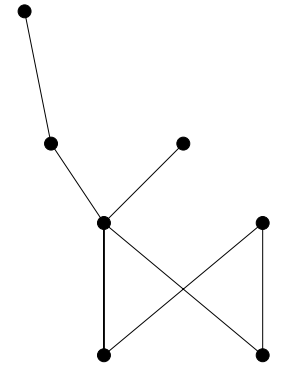

(a)

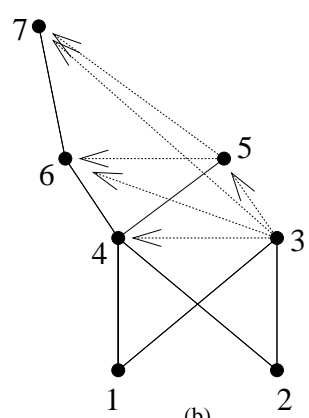

(b)

Figure 5: (a) An element $R \in \mathcal{O}$; (b) The relation $Z$ associated with $R$, where pairs of $Z$ are joined with an arrow. Vertices are then labelled according to a preorder linear extension.

Proposition 2.1 Let $R \in \mathcal{O}$ and suppose that $\lambda_{1}$ and $\lambda_{2}$ are two preorder linear extensions of $R$. If $\lambda_{1}(x)=\lambda_{2}(y)$, then the two elements $x$ and $y$ must be order equivalent in $R$.

Proof. If $\lambda_{1}(x)>\lambda_{1}(y)$ and $\lambda_{2}(x)<\lambda_{2}(y)$, then the thesis follows from the above lemma. Otherwise, without loss of generality, suppose that $\lambda_{1}(x)>\lambda_{1}(y)$ and $\lambda_{2}(x)>\lambda_{2}(y)$. We claim that there exists an element $\tilde{z}$ such that $x$ is equivalent to $\tilde{z}$ which in turn is equivalent to $y$, and this will be enough to conclude.

Indeed, denoting with $X$ the support of $R$, we have that $\mid\{z \in X \backslash\{x, y\}$ : $\left.\lambda_{2}(z)>\lambda_{2}(y)=\lambda_{1}(x)\right\}|+1=|\left\{z \in X \backslash\{x, y\}: \lambda_{1}(z)>\lambda_{2}(y)=\lambda_{1}(x)\right\} \mid$. Then there must exist an element $\tilde{z} \in X \backslash\{x, y\}$ such that $\lambda_{1}(\tilde{z})>\lambda_{1}(x)=\lambda_{2}(y)$ and $\lambda_{2}(\tilde{z})<\lambda_{1}(x)=\lambda_{2}(y)$. Since $\lambda_{1}(\tilde{z})>\lambda_{1}(x)$ and $\lambda_{2}(\tilde{z})<\lambda_{2}(y)<\lambda_{2}(x)$, then $\tilde{z}$ must be order equivalent to $x$ in $R$ (once again thanks to the above lemma). Analogously, since $\lambda_{1}(\tilde{z})>\lambda_{1}(x)>\lambda_{1}(y)$ and $\lambda_{2}(\tilde{z})<\lambda_{2}(y), \tilde{z}$ and $y$ are order equivalent.

Thanks to the above proposition we can assert that, for any $R \in \mathcal{O}$, there is at most one preorder linear extension of $R$. The next proposition shows that indeed a (the) preorder linear extension exists, and also suggests how to find it.

Proposition 2.2 Let $T$ be a planar tree and $\rho$ be the bijection described in section 2. Suppose that the nodes of $T$ are labelled according to the preorder traversal. Then the induced labelling on $\rho(T)$ determines a preorder linear extension of $\rho(T)$.

Proof. With a slight abuse of notation, in this proof we will denote the elements of $T$ and $\rho(T)$ using their labels in the appropriate linear extensions. 
Denote with $<_{t}$ the total order determined by the preorder traversal on $T$. We have to prove that $<_{t}$ is mapped by $\rho$ to a preorder linear extension of $\rho(T)$. According to the definition of preorder linear extension, what we have to show is that, given two nodes $x$ and $y$ of $T$ such that $x<_{t} y$, then the pair $(x, y)$ satisfies the definition of preorder linear extension. More precisely, we must prove that, if $x Z y$ in $\rho(T)$, then necessarily $x<_{t} y$. Indeed, $x Z y$ immediately implies that $x \bar{R} y$. This means that, in $T$, either $x$ is a descendant of $y$ or $y$ is a descendant of $x$. Suppose that the former case holds. The fact that $x Z y$ also implies that there exists an element $z$ in $\rho(T)$ such that $z \bar{R} y$ and $z \bar{R} x$. In particular, this would mean that, in $T, x$ should be a descendant of $z$ and, at the same time, neither $z$ could be a descendant of $y$ nor $y$ could be a descendant of $z$, which is plainly impossible (since $T$ is a tree). Therefore we must have that $y$ is a descendant of $x$ in $T$, and so $x<_{t} y$.

We remark that the definition of preorder linear extension has a meaning only for elements in $\mathcal{O}$ : just observe that it is not possible to construct a linear extension with the required properties neither of $2+2$ nor of the fence of order 4.

In figure 6 an example of the correspondence between preorder traversal and preorder linear extension is shown.

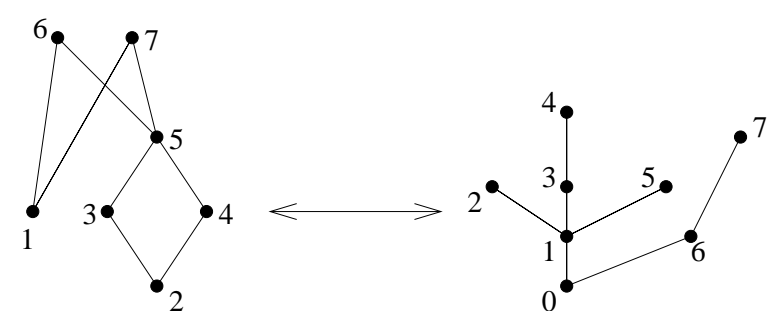

Figure 6: A series parallel interval order with its preorder linear extension and the associated planar tree with the labelling of its node according to the preorder visit.

\section{Catalan lattices on series parallel interval or- ders}

In this section we will define the Dyck lattice and the Tamari lattice on series parallel interval orders whose support is equipped with a preorder linear extension.

In the sequel we will refer to a node of a planar tree using its label in the preorder traversal. Similarly, we will tacitly assume that posets in $\mathcal{O}$ are 
equipped with their preorder linear extension, and we will refer to their elements using the corresponding labels. Moreover, if $x$ and $y$ are labels, we will write $x<y$ referring to the usual order on natural numbers.

Given a planar tree $T, \rho(T)$ will denote the poset obtained through the bijection $\rho$ defined above. In particular, we will often refer to $\rho(T)$ as a binary relation. Given $x \in X$, for a binary relation $B$ on $X$ we consider the set $B(x)=\{y \in X: x B y\}$. If $B$ is a partial order, then the set $B(x)$ is the principal (order) filter generated by the element $x$, whereas $B^{-1}(x)=\{y \in X: y B x\}$ is the principal (order) ideal generated by $x$. In the sequel, we will always use the terms "filter" and "ideal" in place of "order filter" and "order ideal". Moreover, even if this is non standard in poset theory, a principal filter (respectively, ideal) will be considered without its minimum (respectively, maximum).

\subsection{The Dyck lattice}

According to [13, we start by recalling the definition of the Dyck lattice for the set of planar rooted trees with a fixed number of nodes. If $T$ is a planar tree and $k$ is a node of $T$, then define $h_{T}(k)$ as the set of ancestors of $k$ in the tree $T$. Given two planar trees $T_{1}$ and $T_{2}$ having $n$ nodes, $T_{1}$ is less than or equal to $T_{2}$ in the Dyck order, written as $T_{1} \leq_{D} T_{2}$, whenever, for every node $k,\left|h_{T_{1}}(k)\right| \leq\left|h_{T_{2}}(k)\right|$.

The above definition allows us to give a characterization of the Dyck order in terms of series parallel interval orders.

Proposition 3.1 Let $T_{1}, T_{2}$ be two planar trees having $n$ nodes and let $\rho\left(T_{1}\right)=$ $R_{1}$ and $\rho\left(T_{2}\right)=R_{2}$. Then the following conditions are equivalent:

a) $T_{1} \leq_{D} T_{2}$;

b) for every $k,\left|R_{1}^{-1}(k)\right| \geq\left|R_{2}^{-1}(k)\right|$.

Proof. By definition $T_{1} \leq_{D} T_{2}$ if and only if, for all $k,\left|h_{T_{1}}(k)\right| \leq\left|h_{T_{2}}(k)\right|$, which is equivalent to:

$$
\left|\left\{x \in T_{1}: x \notin h_{T_{1}}(k)\right\}\right| \geq\left|\left\{x \in T_{2}: x \notin h_{T_{2}}(k)\right\}\right| .
$$

Consider now the series parallel interval orders $R_{1}$ and $R_{2}$ associated with $T_{1}$ and $T_{2}$ respectively. The previous condition may be expressed by saying that, for all $k$ :

$$
\left|\overline{R_{1}}(k) \cup\left\{x \in T_{1}: x>k\right\}\right| \geq\left|\overline{R_{2}}(k) \cup\left\{x \in T_{2}: x>k\right\}\right| .
$$

To show that (11) is equivalent to $b$ ) observe that, for a generic element $k$, the inequality

$$
\left|\overline{R_{1}}(k) \cup\{k+1, \ldots, n\}\right| \geq\left|\overline{R_{2}}(k) \cup\{k+1, \ldots, n\}\right|
$$

holds if and only if

$$
\left|R_{1}^{-1}(k) \cup\{k+1, \ldots, n\}\right| \geq\left|R_{2}^{-1}(k) \cup\{k+1, \ldots, n\}\right|,
$$


since, for $i=1,2, R_{i}(k) \subseteq\{k+1, \ldots n\}$. Thus, being also $R_{i}^{-1}(k) \cap\{k+$ $1 \ldots, n\}=\emptyset$, we immediately get

$$
\left|R_{1}^{-1}(k)\right|+|\{k+1, \ldots, n\}| \geq\left|R_{2}^{-1}(k)\right|+|\{k+1, \ldots, n\}|,
$$

which is precisely $b$ ).

In figure 7 an application of this proposition is shown. The figure depicts two comparable elements in the Dyck order, and the trees on the left correspond to the posets on the right through $\rho$.

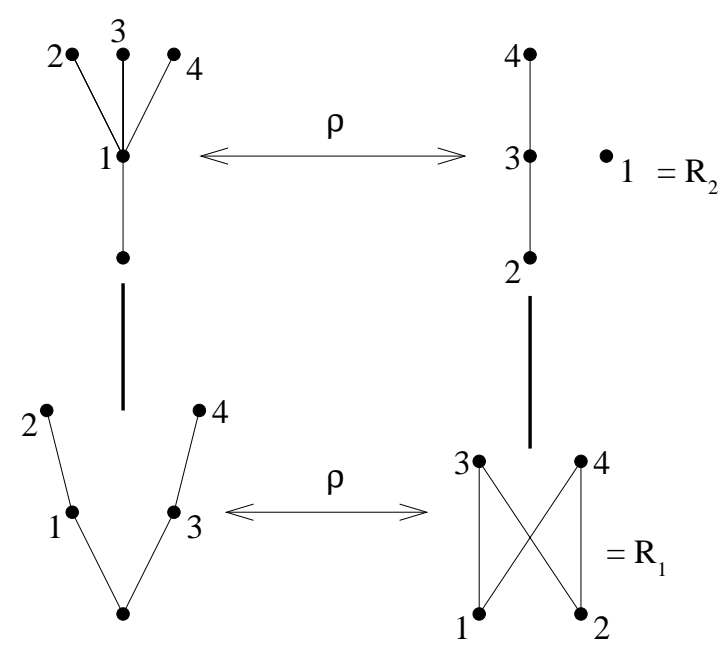

Figure 7: Two comparable elements in the Dyck order.

As a corollary, we find that the Dyck order on series parallel interval orders can be defined in terms of the cardinalities of the principal ideals of these posets.

Corollary 3.1 Given $R_{1}, R_{2} \in \mathcal{O}(n), R_{1} \leq_{D} R_{2}$ if and only if, for every $k \leq n$, $\left|R_{1}^{-1}(k)\right| \geq\left|R_{2}^{-1}(k)\right|$.

See also figure 9 (a) for an example.

\subsection{The Tamari lattice}

According to [13, we start by recalling the definition of the Tamari lattice for the set of planar rooted trees with a fixed number of nodes. If $T$ is a planar tree and $k$ is a node of $T$, then define $u_{T}(k)$ as the set of descendants of $k$ in the tree $T$. Given two planar trees $T_{1}$ and $T_{2}$ having $n$ nodes, $T_{1}$ is less than or 
equal to $T_{2}$ in the Tamari order, written as $T_{1} \leq_{T} T_{2}$, whenever, for every node $k,\left|u_{T_{1}}(k)\right| \leq\left|u_{T_{2}}(k)\right|$.

We provide two equivalent conditions to define the Tamari order on the set of planar rooted trees.

Lemma 3.1 If $T_{1}, T_{2}$ are two planar trees with $n$ nodes, then $T_{1} \leq_{T} T_{2}$ if and only if, for every node $k, u_{T_{1}}(k) \subseteq u_{T_{2}}(k)$.

Proof. Given a node $k$ in the planar tree $T$, if $|u(k)|=j$, then obviously $u(k)=\{k+1, k+2, \ldots, k+j-1, k+j\}$.

Given a node $k$ in the planar tree $T$, consider the set $d(k)=u(k) \cup h(k)$, i.e. the set of ancestors and descendants of $k$. The following lemma holds.

Lemma 3.2 If $T_{1}, T_{2}$ are two planar trees with $n$ nodes, then $u_{T_{1}}(k) \subseteq u_{T_{2}}(k)$ (for every node $k$ ) if and only if $d_{T_{1}}(k) \subseteq d_{T_{2}}(k)$ (for every node $k$ ).

Proof. If $u_{T_{1}}(k) \subseteq u_{T_{2}}(k)$ for all $k$, then $h_{T_{1}}(k) \subseteq h_{T_{2}}(k)$ for all $k$ as well. Indeed, if $x \in h_{T_{1}}(k)$, then $k \in u_{T_{1}}(x)$, hence $k \in u_{T_{2}}(x)$ and so $x \in h_{T_{2}}(k)$. Therefore $d_{T_{1}}(k)=u_{T_{1}}(k) \cup h_{T_{1}}(k) \subseteq u_{T_{2}}(k) \cup h_{T_{2}}(k)=d_{T_{2}}(k)$, for every node $k$.

Now suppose that, for every node $k, d_{T_{1}}(k) \subseteq d_{T_{2}}(k)$. If $x \in u_{T_{1}}(k)$ then $x \in d_{T_{1}}(k)$ and then $x \in d_{T_{2}}(k)$ with $x>k$. So $x \in u_{T_{2}}(k) \cup h_{T_{2}}(k)$, that is $x \in u_{T_{2}}(k)$, since $x>k$.

The above lemma allows us to give the following characterization of the Tamari order.

Proposition 3.2 Let $T_{1}, T_{2}$ be two planar trees having $n$ nodes and let $\rho\left(T_{1}\right)=$ $R_{1}$ and $\rho\left(T_{2}\right)=R_{2}$. Then the following conditions are equivalent:

a) $T_{1} \leq_{T} T_{2}$

b) for every $k, R_{1}(k) \supseteq R_{2}(k)$.

Proof. From the previous lemma we have that $T_{1} \leq_{T} T_{2}$ if and only if, for every $k, d_{T_{1}}(k) \subseteq d_{T_{2}}(k)$. Now $d_{T_{1}}(k) \subseteq d_{T_{2}}(k)$ if and only if, for every $y$, $k R_{2} y$ implies $k R_{1} y$, that is, for every $k, R_{1}(k) \supseteq R_{2}(k)$. Indeed, suppose that, for every $k, R_{1}(k) \supseteq R_{2}(k)$. If $y \notin d_{T_{2}}(k)$ then $k \overline{R_{2}} y$, whence $k \overline{R_{1}} y$, and so $y \notin d_{T_{1}}(k)$. Viceversa, suppose that, for every $k, d_{T_{1}}(k) \subseteq d_{T_{2}}(k)$. If $y \overline{R_{2}} k$, then $y \overline{R_{1}} k$. Now, if $k R_{2} y$, then obviously $k<y$, which cannot hold together with $y R_{1} k$ and so $k R_{1} y$. Thus we can conclude that $y R_{2} k$ implies $y R_{1} k$, as desired.

Figure 8 shows an application of this proposition. The figure depicts two comparable elements in the Tamari order, and the trees on the left correspond to the posets on the right through $\rho$. 
The next lemma shows that the Tamari order may be defined by means of the cardinalities of the principal filters of the posets in $\mathcal{O}$.

Lemma 3.3 Let $T_{1}, T_{2}$ be two planar trees having $n$ nodes and $\rho\left(T_{1}\right)=R_{1}$, $\rho\left(T_{2}\right)=R_{2}$ be the two associated posets in $\mathcal{O}(n)$. Then, for every node $k$, $\left|R_{1}(k)\right| \geq\left|R_{2}(k)\right|$ if and only if $R_{1}(k) \supseteq R_{2}(k)$.

Proof. Just observe that, if $T$ is a planar tree having $n$ nodes, then, for any of its nodes $k, R(k)$ is a final segment of $\{1, \ldots, n\}$.

The above lemma allows us to give another characterization of the Tamari order.

Proposition 3.3 Let $T_{1}, T_{2}$ be two planar trees having $n$ nodes and let $\rho\left(T_{1}\right)=$ $R_{1}$ and $\rho\left(T_{2}\right)=R_{2}$. Then $T_{1} \leq_{T} T_{2}$ if and only if, for every node $k,\left|R_{1}(k)\right| \geq$ $\left|R_{2}(k)\right|$.

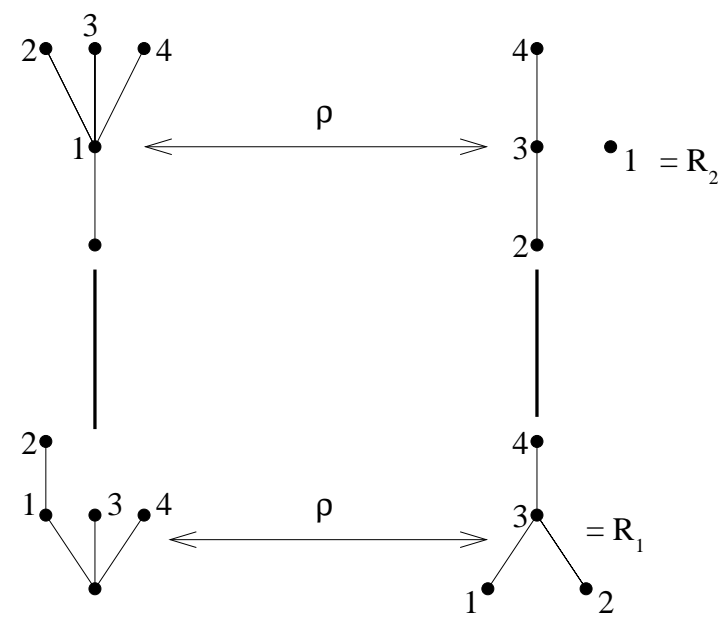

Figure 8: Two comparable elements in the Tamari order.

As a corollary of the above propositions, we can immediately obtain a definition of the Tamari order on series parallel interval orders in terms of the principal filters of these posets.

Corollary 3.2 Given $R_{1}, R_{2} \in \mathcal{O}(n), R_{1} \leq_{T} R_{2}$ if and only if, for every $k \leq n$, $\left|R_{1}(k)\right| \geq\left|R_{2}(k)\right|$. Equivalently, $R_{1} \leq_{T} R_{2}$ if and only if, for every $k \leq n$, $R_{1}(k) \supseteq R_{2}(k)$. 
In figure 9(b) the Tamari lattice on the five elements belonging to $\mathcal{O}(3)$ is depicted .

Remark. We know from 13 that the Dyck and the Tamari orders are related by the following refinement property: given two Catalan structures $T_{1}, T_{2}$ of the same size, if $T_{1} \leq_{T} T_{2}$ then $T_{1} \leq_{D} T_{2}$. This fact is an obvious consequence of Corollaries 3.1 and 3.2 . Our approach seems to be particulary interesting since it is now possible to prove such a refinement property in a very neat way. Indeed, if $R_{1}(k) \supseteq R_{2}(k)$ holds for any $k$, then we also have that, for all $k$, $R_{1}^{-1}(k) \supseteq R_{2}^{-1}(k)$, and so, for all $k,\left|R_{1}^{-1}(k)\right| \geq\left|R_{2}^{-1}(k)\right|$.

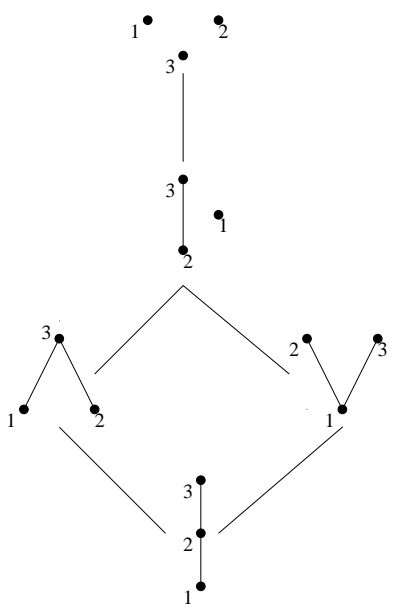

(a)

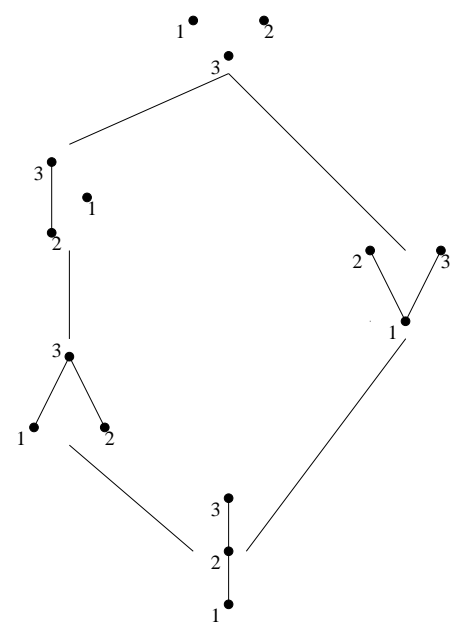

(b)

Figure 9: The two Catalan lattices defined on the class of series parallel interval orders of size three: (a)The Dyck lattice (b)The Tamari lattice.

\section{Series parallel interval orders and pattern avoid- ing permutations}

The strong Bruhat order $\left(\leq_{B}\right)$ and the weak Bruhat order $\left(\leq_{b}\right)$ are two well known partial orders defined on the set of permutations having fixed length [16. We briefly recall here their definitions.

Given a permutation $\pi=a_{1} a_{2} \ldots a_{n}$, a reduction of $\pi$ is a permutation obtained from $\pi$ by interchanging some $a_{i}$ with some $a_{j}$, provided that $i<j$ and $a_{i}>a_{j}$. We say that $\pi_{1}<_{B} \pi_{2}$ whenever $\pi_{1}$ is obtained from $\pi_{2}$ through a sequence of reductions. Define a simple reduction of $\pi=a_{1} a_{2} \ldots a_{n}$ as a permutation obtained from $\pi$ by interchanging some $a_{i}$ with some $a_{i+1}$, provided that 
$a_{i}>a_{i+1}$. We say that $\pi_{1}<_{b} \pi_{2}$ whenever $\pi_{1}$ is obtained from $\pi_{2}$ through a sequence of simple reductions.

In this section we will consider another well known Catalan structure, namely the class of permutations avoiding the pattern 312 , and we will prove, using a characterization given in [1, that the strong Bruhat order, when restricted to such a class of pattern avoiding permutations, is isomorphic to the Dyck order. Moreover we will show that an analogous isomorphism also exists between the Tamari lattice and the weak Bruhat order on the same class of permutations. We remark that these two results have been already obtained independently in 1] (for the Dyck case) and in [6] (for the Tamari case; see also [8]). Here our main aim is to find a common language for these two results.

We start by describing a bijection between series parallel interval orders on $n$ elements and permutations of length $n$ avoiding the pattern 312, denoted by $A v_{n}(312)$. Our approach can be compared with the one used in [5] to enumerate posets avoiding $2+2$.

First of all recall that the set of principal filters of a poset avoiding $2+2$ is linearly ordered by inclusion. The interested reader can find a proof of this fact in [9, where it is also proved that this condition completely characterizes such a class of posets.

Given a poset $R \in \mathcal{O}(n)$, consider the labelling of its elements determined by its preorder linear extension and denote its principal filters by $R(1), R(2)$, $\ldots, R(n)$. Define a permutation $\pi=\pi(R)$ of length $n$ as follows: $k$ precedes $j$ in $\pi$ precisely when either $R(k) \supset R(j)$ or $R(k)=R(j)$ and $k>j$. It is easy to show that, for each $R \in \mathcal{O}, \pi(R)$ does not contain the pattern 312, and the function $R \mapsto \pi(R)$ is a bijection between $\mathcal{O}(n)$ and $A v_{n}(312)$.

Remark. Observe that our bijection cannot be described in terms of principal ideals (instead of principal filters), due to our choice of taking the preorder linear extension of a poset.

For instance, the permutation associated with the poset depicted in figure 10 is 2146753. Indeed the filters of such a poset are $R(1)=R(2)=\{3,4,5,6,7\}$, $R(3)=R(5)=R(7)=\emptyset, R(4)=\{5,6,7\}, R(6)=\{7\}$ and then they are listed as follows:

$$
R(2)=R(1) \supset R(4) \supset R(6) \supset R(7)=R(5)=R(3) .
$$

Remark. Given a permutation $\pi=a_{1} \cdots a_{n}$ of length $n$ and the partial order relation $R \in \mathcal{O}(n)$ associated with it, it is not difficult to observe that $R\left(a_{j}\right)$ is the set of the elements of $\pi$ greater than $a_{j}$ and following $a_{j}$ in $\pi$. Analogously, $R^{-1}\left(a_{j}\right)$ is the set of the elements of $\pi$ lesser than $a_{j}$ and preceding $a_{j}$ in $\pi$ (see again figure 100). In what follows, we will use the notations $f_{\pi}\left(a_{j}\right)$ and $i_{\pi}\left(a_{j}\right)$ in place of $R\left(a_{j}\right)$ and $R^{-1}\left(a_{j}\right)$ (respectively) when dealing with permutations rather than partial order relations. 


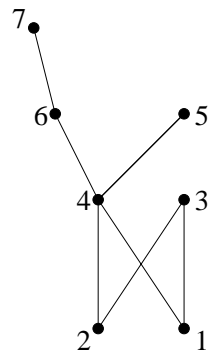

Figure 10: A series parallel interval order and its preorder extension.

\subsection{The Tamari lattice and the weak Bruhat order on Av(312)}

According to [12, it is possible to characterize the weak Bruhat order on permutations using inversions. Recall that an inversion of $\pi=a_{1} a_{2} \ldots a_{n}$ is a pair $\left(a_{i}, a_{j}\right)$ such that $i<j$ and $a_{i}>a_{j}$. Given two permutations of the same length $\pi_{1}$ and $\pi_{2}$, it is $\pi_{1} \leq_{b} \pi_{2}$ if and only if the set $E\left(\pi_{1}\right)$ of inversions of $\pi_{1}$ is a subset of the set $E\left(\pi_{2}\right)$ of inversion of $\pi_{2}$. The following simple proposition provides the key ingredient to prove that the Tamari lattice is isomorphic to the weak Bruhat order on $A v(312)$.

Proposition 4.1 Let $\pi_{1}$ and $\pi_{2}$ be two permutations of length $n$. Then $E\left(\pi_{1}\right) \subseteq$ $E\left(\pi_{2}\right)$ if and only if, for every $1 \leq k \leq n, f_{\pi_{1}}(k) \supseteq f_{\pi_{2}}(k)$.

Proof. Suppose that, for every $k, f_{\pi_{1}}(k) \supseteq f_{\pi_{2}}(k)$. If $(i, j) \in E\left(\pi_{1}\right)$, with $i>j$, then $i \notin f_{\pi_{1}}(j)$ and then $i \notin f_{\pi_{2}}(j)$. This implies that $(i, j) \in E\left(\pi_{2}\right)$.

Viceversa, suppose that $E\left(\pi_{1}\right) \subseteq E\left(\pi_{2}\right)$. If $i \in f_{\pi_{2}}(j)$, then $(i, j) \notin E\left(\pi_{2}\right)$, whence $(i, j) \notin E\left(\pi_{1}\right)$. This implies that $i \in f_{\pi_{1}}(j)$.

Corollary 4.1 The Tamari order is isomorphic to the weak Bruhat order restricted to $A v_{n}(312)$.

\subsection{The Dyck lattice and the strong Bruhat order on Av(312)}

For a given permutation $\pi$ of length $n$, define the vector $\max _{\pi}$ as follows: $\max _{\pi}(k)=\max \{\pi(i): i \leq k\}$. According to [1, we recall that, given two 312-avoiding permutations $\pi_{1}$ and $\pi_{2}$ of length $n, \pi_{1} \leq_{B} \pi_{2}$ if and only if, for all $1 \leq k \leq n, \max _{\pi_{1}}(k) \leq \max _{\pi_{2}}(k)$. For instance, considering the two permutations $\pi_{1}=468753921, \pi_{2}=768543921 \in A v_{9}(312)$, we have $\max _{\pi_{1}}=$ $(4,6,8,8,8,8,9,9,9)$ and $\max _{\pi_{2}}=(7,7,8,8,8,8,9,9,9)$, whence $\pi_{1} \leq_{B} \pi_{2}$. Indeed, starting from $\pi_{2}$, we obtain $\pi_{1}$ by the following reductions: $\pi_{2}=$ 
$768543921 \rightarrow 768453921 \rightarrow 468753921=\pi_{1}$. Observe that, in the above sequence of reductions, the permutation 768453921 is not 312 -avoiding.

Given a permutation $\pi$ of length $n$, consider the set of its consecutive noninversions, i.e. the set of all $m \in\{1, \ldots, n\}$ such that either $m=n$ or the pair $(m, m+1)$ is a noninversion of $\pi$ (that is $m$ appears before $m+1$ in $\pi$ ). The following lemma provides a characterization of consecutive noninversions in permutations avoiding 312 .

Lemma 4.1 Let $\pi \in A v_{n}(312)$, then the following properties hold:

i) $m$ is a consecutive noninversion of $\pi$ if and only if either $m=n$ or $\left|i_{\pi}(m)\right| \neq\left|i_{\pi}(m+1)\right| ;$

ii) if $j<k$, then $\left|i_{\pi}(j)\right| \leq\left|i_{\pi}(k)\right|$;

iii) if $k=\left|i_{\pi}(m)\right|$ and $m$ is a consecutive noninversion of $\pi$, then $\pi(k+1)=m$;

iv) the consecutive noninversions of $\pi$ are those elements of $\pi$ preceded only by lesser entries;

$v$ ) the set of all consecutive noninversions of $\pi$ coincides with the set of components of $\max _{\pi}$. Moreover, the index of the consecutive noninversion $m$ in $\pi$ coincides with the index of the first occurrence of $m$ in $\max _{\pi}$.

Proof. i) If $m \neq n$ is a consecutive noninversion, then obviously $\mid i_{\pi}(m+$ $1)|\geq| i_{\pi}(m) \mid-1$, whence $\left|i_{\pi}(m)\right| \neq\left|i_{\pi}(m+1)\right|$. Viceversa, suppose that $m \neq n$ is such that $\left|i_{\pi}(m)\right| \neq\left|i_{\pi}(m+1)\right|$; if $(m+1, m)$ were an inversion of $\pi$, then there should be an entry $j<m$ between $m+1$ and $m$, which is impossible since $\pi \in A v_{n}(312)$.

ii) This is an immediate consequence of the fact that $\pi \in A v_{n}(312)$.

iii) This is obvious when $m=n$. Otherwise, suppose that $m$ is an element of $\pi$ having more than $i$ elements on its left; then there would be at least one element $j$ such that $j$ precedes $m$ and $j>m$, and the three elements $j, m, m+1$ would show a 312-pattern in $\pi$, a contradiction.

iv) Observe that, if $m$ is a consecutive noninversion of $\pi$, then, by iii), all elements of $\pi$ preceding $m$ are less than $m$. Viceversa, if $m$ is an entry of $\pi$ preceded only by lesser elements, one cannot have $\left|i_{\pi}(m)\right|=\left|i_{\pi}(m+1)\right|$, since in this case $m+1$ would precede $m$. Then $m=n$ or $\left|i_{\pi}(m)\right|<\left|i_{\pi}(m+1)\right|$.

$v$ ) This is a direct consequence of $i v)$.

Proposition 4.2 Given $\pi_{1}, \pi_{2} \in A v_{n}(312)$, the following conditions are equivalent:

a) for every $1 \leq k \leq n,\left|i_{\pi_{1}}(k)\right| \geq\left|i_{\pi_{2}}(k)\right|$;

b) for every $1 \leq k \leq n, \max _{\pi_{1}}(k) \leq \max _{\pi_{2}}(k)$. 
Proof. $a) \Rightarrow b$ ) Let $j^{\prime}=\pi_{1}(j)$ be a consecutive noninversion of $\pi_{1}$. Thanks to the above lemma, item iii), all the elements of $\pi_{1}$ preceding $j^{\prime}$ are less than $j^{\prime}$. We claim that there exists $k=\pi_{2}(h)$ such that $k>j^{\prime}, h \leq j$ and all the elements of $\pi_{2}$ before $k$ are less than $k$. Indeed, we have $j-1=\left|i_{\pi_{1}}\left(j^{\prime}\right)\right| \geq$ $\left|i_{\pi_{2}}\left(j^{\prime}\right)\right|=\left|i_{\pi_{2}}(k)\right|=t$, where $k$ is the first consecutive noninversion of $\pi_{2}$ on the left of $j^{\prime}$ with $k \geq j^{\prime}$ (such a $k$ does indeed exist, as the reader can immediately check). Then $k=\pi_{2}(t+1)$ is the desired element of $\pi_{2}$. As a consequence, we have that, if $j^{\prime}$ is a consecutive noninversion of $\pi_{1}$, then $\max _{\pi_{1}}\left(j^{\prime}\right) \leq \max _{\pi_{2}}\left(j^{\prime}\right)$. From this we can immediately deduce the same inequality for any $j^{\prime} \leq n$.

$b) \Rightarrow a$ ) Set $t=\left|i_{\pi_{1}}(j)\right|$, consider the consecutive noninversion $j^{\prime}$ of $\pi_{1}$ such that $\left|i_{\pi_{1}}\left(j^{\prime}\right)\right|=t$ with $j^{\prime} \geq j$ (this is the first consecutive noninversion of $\pi_{1}$ on the left of $j$ ). From the previous lemma we have $j^{\prime}=\max _{\pi_{1}}(t+1) \leq \max _{\pi_{2}}(t+$ $1)=\max _{\pi_{2}}(h)$, where $h \leq t+1$ is the index of the first component of $\max _{\pi_{2}}$, which is equal to $\max _{\pi_{2}}(t+1)$. Again from the previous lemma, we have that $\max _{\pi_{2}}(h)=k$ is a consecutive noninversion of $\pi_{2}$ and $\left|i_{\pi_{2}}(k)\right|=h-1$. Finally, using in particular item ii) of the above lemma in the first two inequalities, we have :

$$
\left|i_{\pi_{2}}(j)\right| \leq\left|i_{\pi_{2}}\left(j^{\prime}\right)\right| \leq\left|i_{\pi_{2}}(k)\right|=h-1 \leq t=\left|i_{\pi_{1}}(j)\right| .
$$

Corollary 4.2 The Dyck order is isomorphic to the strong Bruhat order restricted to $A v_{n}(312)$.

\section{$5 \quad$ Further works}

In the present work we have considered two well known Catalan posets and we have proposed a unifying language to describe them based on the notion of series parallel interval order. There are of course several other poset structures which can be considered on the objects of the Catalan family. Maybe the most famous one is the Kreweras order [14, which is naturally defined on noncrossing partitions of a set of given cardinality by refining the classical partial order on set partition. Other less classical posets have been defined by Baril and Pallo on Dyck words (the phagocyte lattice [2]) and on binary trees (the pruning-grafting lattice [3]). It seems natural to ask if series parallel interval orders can be used also to describe these (and maybe other) Catalan posets. Unfortunately, we have not been able to find an answer to such a question yet.

\section{References}

[1] E. Barcucci, A. Bernini, L. Ferrari, M. Poneti, A distributive lattice structure connecting Dyck paths, noncrossing partitions and 312-avoiding permutations, Order, 22 (2005) 311-328. 
[2] J. L. Baril, J. M. Pallo, The phagocyte lattice of Dyck words, Order, 23 (2006) 97-107.

[3] J. L. Baril, J. M. Pallo, The pruning-grafting lattice of binary trees, Theoret. Comput. Sci., 409 (2008) 382-393.

[4] O. Bernardi, N. Bonichon, Intervals in Catalan lattices and realizers of triangulations, J. Combin. Theory Ser. A, 116 (2009) 55-75.

[5] M. Bousquet-Melou, A. Claesson, M. Dukes, S. Kitaev, Unlabeled (2+2)free posets, ascent sequences and pattern avoiding permutations, J. Combin. Theory Ser. A, to appear (2010).

[6] A. Björner, M. Wachs, Shellable nonpure complexes and posets 2, Trans. Am. Math. Soc., 349 (1997) 3945-3975.

[7] F. Disanto, L. Ferrari, R. Pinzani, S. Rinaldi, Catalan pairs: A relationaltheoretic approach to Catalan numbers, Adv. Appl. Math., to appear (2010).

[8] B. Drake, The weak order on pattern-avoiding permutations, proceedings of FPSAC 2005, Taormina (Italy).

[9] M. H. El-Zahar, Enumeration of ordered sets, I. Rival (Ed.), Algorithms and order, Kluwer Acad. Publ., Dordrecht, 1989, pp.327-352.

[10] P. C. Fishburn, Intransitive indifference with unequal indifference intervals, J. Mathematical Psychology, 7 (1970) 144-149.

[11] S. Huang, D. Tamari, Problems of associativity: A simple proof of the lattice property of systems ordered by a semi-associative law, J. Combin. Theory Ser. A, 13 (1972) 7-13.

[12] D. Knuth, The art of computer programming, Vol. 3, Addison Wesley, 1998.

[13] D. Knuth, The art of computer programming, Vol. 4, Addison Wesley, 2006.

[14] G. Kreweras, Sur les partitions non croisées d'un cycle, Discrete Math., 1 (1972) 333-350.

[15] J. P. S. Kung, G.-C. Rota, C. H. Yan, Combinatorics: The Rota Way, Cambridge University Press, Cambridge, 2009.

[16] R. P. Stanley, Enumerative Combinatorics, Vol. 1, Cambridge University Press, 1997.

[17] R. P. Stanley, Enumerative Combinatorics, Vol. 2, Cambridge University Press, 1999. 